\title{
The effect of enamel matrix derivatives on root coverage: a 12-month follow-up of a randomized clinical trial
}

\author{
Hacer Sahin AYDINYURT ${ }^{(a)}$ \\ Yasin TEKIN(b) iD
}

Abdullah Seckin ERTUGRUL(

(a) Van Yuzuncu Yil University, Faculty of Dentistry, Department of Periodontology, Van, Turkey.

(b) Trakya University, Faculty of Dentistry, Department of Periodontology, Edirne, Turkey

(c) Izmir Katip Çelebi University, Faculty of Dentistry, Department of Periodontology, Izmir Turkey

Declaration of Interests: The authors certify that they have no commercial or associative interest that represents a conflict of interest in connection with the manuscript.

\section{Corresponding Author:}

Hacer Sahin Aydınyurt

E-mail:dthacersah@hotmail.com,

hacersahinay@gmail.com.tr

Submitted: March 29, 2018

Accepted for publication: November 26, 2018

Last revision: December 06, 2018
Abstract: Subepithelial connective tissue grafts (SCTGs) with a coronally advanced flap (CAF) are accepted as the gold standard for covering denuded root surfaces. In recent years, enamel matrix derivatives (EMDs) have been used for their regenerative potential in periodontics. The aim of this split-mouth and randomized controlled study was to assess the clinical and aesthetical impacts of EMD application in combination with SCTG+CAF in patients with Miller's Class I and II gingival recessions in contralateral canines of the maxilla. Participants who underwent SCTG+CAF+EMD application were identified as the test group $(n=19)$ and those who underwent SCTG+CAF as control group $(n=19)$. The outcome parameters were recession depth/width, root coverage percentage, and root coverage aesthetic score (RES). RES was evaluated by two calibrated blind periodontists one year after the treatment. Statistically significant root coverage percentage was observed at one year post-treatment for both groups $(p<0.05)$. However, significant differences between the groups were not observed in terms of total RES and complete root coverage rate $(p>0.05)$. The test group had significantly better results than the control according to the soft tissue texture and mucogingival junction alignment results $(\mathrm{p}<0.05)$. These results indicate that EMDs contribute to the healing of soft tissue without scarring. As a result of better wound healing, the EMD-added group exhibited better results in terms of the harmony of the mucogingival junction between adjacent teeth. This paper is the first split-mouth study in which SCTG+CAF and $\mathrm{SCTG}+\mathrm{CAF}+\mathrm{EMD}$ were compared using RES in bilateral canines.

Keywords: Gingival Recession; Autrografts; Periodontics.

\section{Introduction}

The migration of the gingival margin to the apex is defined as gingival recession. ${ }^{1}$ Gingival recession can occur in single or multiple teeth. $^{2}$ A common disease, it was reported to have an incidence of $88 \%$ among individuals aged 65 years. ${ }^{3}$ Gingival recession may cause dentin sensitivity and aesthetic concerns in patients. ${ }^{4}$ Subepithelial connective tissue grafts (SCTGs) with a coronally advanced flap (CAF) are accepted as the gold standard for covering denuded root surfaces in periodontics. ${ }^{5}$ 
Another approach used to improve the treatment efficacy of root coverage is the application of enamel matrix protein derivatives (EMDs). ${ }^{6,7}$ EMDs are potential regenerative materials and their contents serve as proteins capable of inducing the formation of new periodontal ligament fibres, cementum, and alveolar bone. ${ }^{7}$ Amelogenin, the protein responsible for the biological activity of EMDs, accounts for $90 \%$ of the total EMD content. ${ }^{8,9}$ Because of their role in cementum development, EMDs are thought to induce regeneration of periodontal tissues. ${ }^{10,11,12}$ It was reported that EMDs have a positive effect on cell proliferation and survival, cell adhesion, spreading and chemotaxis, and the expression of transcription factors, growth factors, cytokines, extracellular matrix components, and other macromolecules. ${ }^{13}$ Clinical indications of EMDs include nonsurgical periodontal therapy, guided bone regeneration, bone defects, furcation defects, and gingival recession treatments. In addition, it was suggested that EMDs improves early wound healing.?

For denuded root surfaces, the ideal result is the covering of the root surface with soft tissue-compatible material that cannot be distinguished from adjacent tissue. ${ }^{14}$ Clinical results of root coverage are generally evaluated according to the root coverage percentage and sometimes the complete root coverage ${ }^{15}$ Cairo et al. ${ }^{16}$ showed that evaluating only the gingival margin level after surgical treatment does not fully reflect the aesthetic results. However, even if complete root surface coverage is ensured in some cases, aesthetic expectations are not fully met due to different tissue thicknesses or scar tissue formation. Root coverage aesthetic scoring (RES) is an improved method for subjective assessment of the treatment success of gingival recession. The RES scale evaluates at the same time the gingival margin, marginal tissue contour, soft tissue texture, mucogingival junction alignment, and gingival color. ${ }^{16}$ In light of this information, the null hypothesis of this study was that the combination of EMDs with SCTG+CAF does not have an impact on aesthetic and clinical success of gingival recession treatment. The aim of this study was to assess the clinical and aesthetic impact of EMD application in combination with SCTG+CAF in bilateral canine Miller class I or II gingival recessions.

\section{Methodology}

\section{Patient selection}

In this split-mouth, randomized controlled study, 38 bilateral canine teeth from 19 patients aged 18-55 years were included. Patients consulted with complaints of poor aesthetic and / or root surface hypersensitivity to the Department of Periodontology of the Faculty of Dentistry at Van Yuzuncu Yil University. The patients included in this study had no systemic disease, did not smoke cigarettes or use tobacco products, and were not in a pregnancy and breastfeeding period; they had class I or class II gingival recession according to the Miller gingival classification, had a recession depth of $\geq 2 \mathrm{~mm}$, were without a restoration or caries, and had not undergone an operation in the relevant dental region.

All the study's methods were approved by the ethics committee at Van Yuzuncu Yil University (B.30.2.YYU.0.01.00.00/32). This study was also approved at clinicaltrials.gov (NCT03197610). After the enrollment was completed, the study protocol was explained to all patients and written consent forms were signed by all participants.

\section{Study population}

The study included 19 patients (10 females and 9 males) who met the inclusion criteria. In the control group and the test group, 19 isolated single-tooth defects were treated in maxillary canine teeth. In the test group, 10 class I and 9 class II recession defects were treated. In the control group, 9 class I and 10 class II recession defects were treated. The treatment modalities were described to all the patients, but the participants were blind regarding which tooth was in the test group and which was in the control group.

\section{Sample size}

Before starting the study, the number of patients was determined by statistical power analysis. A difference of $1 \mathrm{~mm}$ in gingival recession height was considered the minimum significant difference. With an alpha of 0.05 and power of $80 \%$ the sample size of 14 was calculated. In calculations, the sigma value $(0.80 \mathrm{~mm})$ indicated in previous studies was used..$^{6,17,18}$ 


\section{Randomization}

In this study, a simple randomization technique, the coin flipping method, was used. For each right canine a coin was flipped. The side of the coin determined the group assignment of each right canine tooth (heads: control; tails: treatment). The left canine was included in the remaining group.

\section{Initial therapy}

After enrolment, and one month before the surgery, all participants were informed about possible causes of gingival recession. Oral hygiene instructions were explained at the beginning of phase- 1 treatment. After scaling and root planning, the patients who had bleeding index of less than $15 \%$ received the surgical phases. In addition, after the phase- 1 treatment, teeth with periodontal pocket depth of over $3 \mathrm{~mm}$ were not included in surgical phases. These teeth were maintained in phase- 1 therapy and then received the surgical phases.

\section{Surgical procedures}

After local anesthesia was applied to the operation area, the denuded root surface was planned with periodontal curette. The surface of the root was thoroughly washed with saline solution to remove cement residues from the area. The Langer and Langer technique was used to prepare the recipient side. The labial surfaces of adjacent interdental papillae were de-epithelialized. The graft dimensions were measured with periodontal probes, and they exceeded the dimensions of the defect area by at least $1 \mathrm{~mm}$. The anesthetic solution was applied to the donor site in the palatal region on the same side as the operation site. The dimensions of the recipient area were measured by periodontal probes, four bleeding centers were created in the palatal region, and the connective tissue graft was then removed. In order to control the bleeding in the donor area pressure was applied with $0.9 \%$ saline-impregnated sponges. The graft was placed on the recipient site and fixed with 6.0 resorbable sutures, and the flap was fixed with 4.0 silk sutures. Pressure was applied to the operation area for five minutes with saline impregnated sponges.

Gingival recessions in the control group were treated with SCTG+CAF; the EMD selected for this study (Emdogain ${ }^{\circledR}$, Straumann, Basel, Switzerland) was used in addition to SCTG+CAF only in the test group. Prior to EMD application, 24\% EDTA (PrefGel, Straumann, Basel, Switzerland) was applied to the root surface for two minutes. The area was washed with saline and EMD was then applied. Postoperative recommendations were made to the patient. Antibiotics, analgesics, and mouthwashes were prescribed. All surgical procedures were performed by a single periodontist (H.S.A). Sutures were removed in 10-14 days.

\section{Root coverage aesthetic score and clinical measurements}

The RES system was developed by Cairo et al.. ${ }^{16}$ Each tooth was scored by two periodontists who were unaware of the treatment methods. Fifteen days before the study, examiners were calibrated; they scored 20 images and inter-examiner agreement was evaluated. After 15 days examiners re-scored the same 20 images and intra-examiner agreement was evaluated. There are five parameters in the RES. The first is gingival marginal (GM) scored as 0,3 or 6, as follows; unsuccessful root surface closure (0), partial closure (3), and complete root surface closure (6). The second is marginal tissue contour (MTC), scored as irregular (0, not following the cemento-enamel junction) or regular (1) (following the cemento-enamel junction). If there was scar formation, soft tissue texture (STT) was scored as 0 and no scar formation scored as 1 . Mucogingival junction alignment (MJA) was assigned a score of 0 if it was not aligned with the mucogingival junction of the adjacent tooth and a score of 1 if it was aligned with the mucogingival junction of the adjacent tooth. On the gingival color scale (GC), a score of 0 was assigned if the color was different that the adjacent tooth gingival color, and a score of 1 was assigned if the gingival color was similar to the adjacent soft tissue (Table 1). The average score was calculated by the mean scores of two raters. The worst overall RES score was 0 and the best was 10 .

Gingival recession height (GRH) was measure as the distance between the gingival margin and the free gingival margin, and gingival recession width (GRW) as the distance between the two sides 
The effect of enamel matrix derivatives on root coverage: a 12-month follow-up of a randomized clinical trial

Table 1. Root coverage aestehtic scoring system (developed by Cairo et al. ${ }^{16}$ ).

\begin{tabular}{|c|c|c|c|c|}
\hline Variable & 0 point & 1 point & 3 points & 6 points \\
\hline Gingival margin & Failed & $x$ & Partial & Complete \\
\hline Marginal tissue contour & Irregular & Proper & $x$ & $x$ \\
\hline Soft tissue texture & Scar formation present & Scar formation not present & $x$ & $x$ \\
\hline $\begin{array}{l}\text { Mucogingival junction } \\
\text { alignment }\end{array}$ & $\begin{array}{c}\text { Not aligned with the MGJ of adjacent } \\
\text { teeth }\end{array}$ & Aligned with the MGJ of adjacent teeth & $x$ & $x$ \\
\hline Gingival color & Integrated with the adjacent soft tissues & Not integrated with adjacent soft tissues & $x$ & $x$ \\
\hline
\end{tabular}

Table 2. Gingival recession height, gingival recession width, RES scores, and mean RES (tO: pre-treatment, $t 1$ : after 1 year of treatment).

\begin{tabular}{lccc}
\hline Variable & Test group & Control group & p-value \\
\hline Gingival recession height & & & 0.08 \\
+0 & $3.91 \pm 1.20$ & $4.04 \pm 1.42$ & 0.07 \\
+1 & $1.34 \pm 1.11$ & $1.91 \pm 1.56$ & 0.1 \\
Gingival recession width & & & 0.1 \\
+0 & $3.73 \pm 0.91$ & $3.73 \pm 0.75$ & $2.21 \pm 1.62$ \\
+1 & $2.00 \pm 1.41$ & & 0.07 \\
RES Scores & & $4.68 \pm 1.53$ & 0.12 \\
Gingival margin & $5.0 \pm 1.46$ & $0.98 \pm 1.25$ & $0.03^{*}$ \\
Marginal tissue contour & 1.0 & $0.81 \pm 0.4^{*}$ & $0.03^{*}$ \\
Soft tissue texture & $0.93 \pm 0.25^{*}$ & $0.87 \pm 0.34^{*}$ & 0.06 \\
Mucogingival junction alignment & $1.0^{*}$ & $0.92 \pm 0.97$ & 0.07 \\
Gingival colour & 1.0 & $8.37 \pm 1.85$ & \\
Mean RES & $8.93 \pm 1.43$ & & \\
\hline
\end{tabular}

${ }^{*} p<0.05$ : statistically significant.

of the recession defects. The root coverage rate was calculated with the following formula: (baseline GRH - first year GRH) / baseline GRH).

\section{Statistical analysis}

The Kolmogorov-Smirnov normality test was used in the obtained data. Since the data were not normally distributed, the results in the test and control groups were evaluated using the Mann-Whitney $U$ test. The Wilcoxon test was used for evaluation of GRH and GRW data before and after treatment. Cohesion coefficients between the observers were calculated using kappa analysis.

\section{Results}

A total of 38 gingival recession sites in 19 patients (10 females and 9 males) were investigated in this randomized, split-mouth controlled trial. The mean age of patients was $32.18 \pm 8.91$ years. There was no statistically significant difference between groups in terms of average age $(p>0.05)$. In the control group, 19 isolated single gingival recessions (9 Miller class I, 10 Miller class II) were treated with SCTG+CAF. In the test group, 19 isolated single gingival recessions (10 Miller class I, 9 Miller class II) were treated with $\mathrm{SCTG}+\mathrm{CAF}+\mathrm{EMD}$. All gingival recessions were single and isolated in maxillary canine teeth. In total, 50\% of the recessions were Miller class I and 50\% were Miller class II. No statistically significant difference between groups in terms of defect characteristics were observed $(p>0.05)$.

There was no statistically significant difference between initial values of GRH and GRW in the test and control groups $(p>0.05)$. There was a statistically significant decrease one year after 

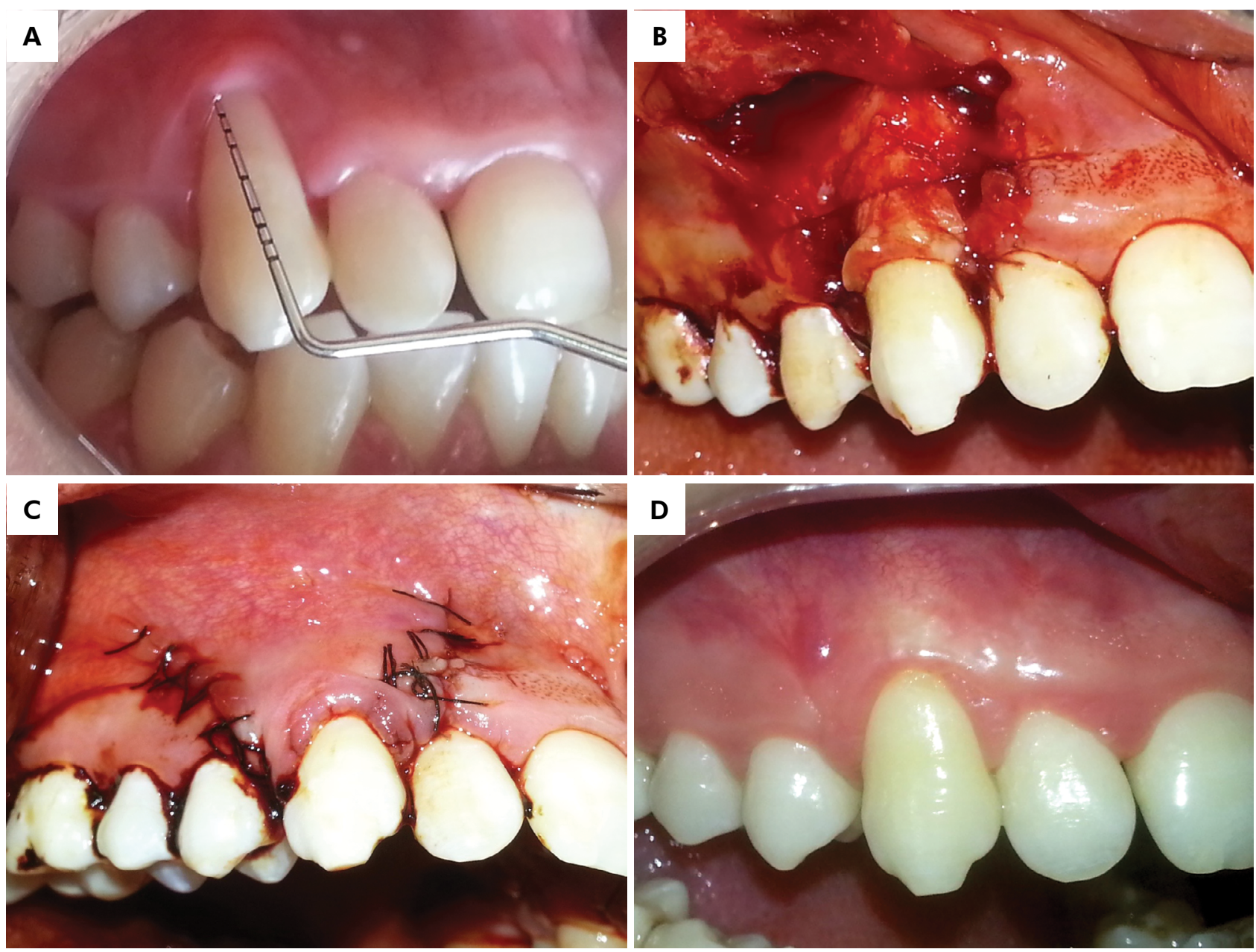

Figure 1. A. Gingival recession in right canine; B. EMD application and SCTG placement; C. Suturing; D. One year post-operative;

treatment in both groups compared to baseline values $(\mathrm{p}<0.05)($ Table 2$)$.

GRW values exhibited a statistically significant decrease 12 months after the surgeries in both groups $(p<0.05)$. There was no difference between the two groups in terms of decrease of GRH and GRW values ( $p$ $>0.05$ ). The complete root coverage rate was calculated as $68 \%$ in the test group and $52 \%$ in the control group. For teeth used in the present study, agreement between the two periodontists who performed RES was evaluated by kappa analysis, which indicated that there was high agreement between the raters for each parameter (GM, MTC, STT, MJA, GC) evaluated $(p<0.05)$. The kappa coefficient was calculated as 1 for GC, 0.445 for MTC, 0.445 for STT, 0.642 for MJA, and 0.721 for GC between the two raters. In addition, there was statistically significant agreement between the raters in terms of total RES scores obtained from root surface aesthetic scoring ( $\mathrm{p}<0.05$ ).

Based on the aesthetic score of root coverage described by Cairo (2009), mean GM, MTC, STT, MJA, and GC scores are exhibited in Table 2. There was no significant difference between the test and control groups in terms of GM, MTC, and GC scores $(p>0.05)$. However, a significant difference was found between the two groups in terms of the mean values of STT $(p<0.05)$ and in MJA scores ( $p<0.05)$.

The root coverage aesthetic score obtained with the sum of GM, MTC, STT, MJA, and GC scores was $8.93 \pm 1.43$ in the test group and $8.37 \pm 1.85$ in the control group (Table 2) but this difference was not statistically significant $(\mathrm{p}>0.05)$.

There were 18 teeth in the test group and 17 in the control group with a GM score of 6 and a root 

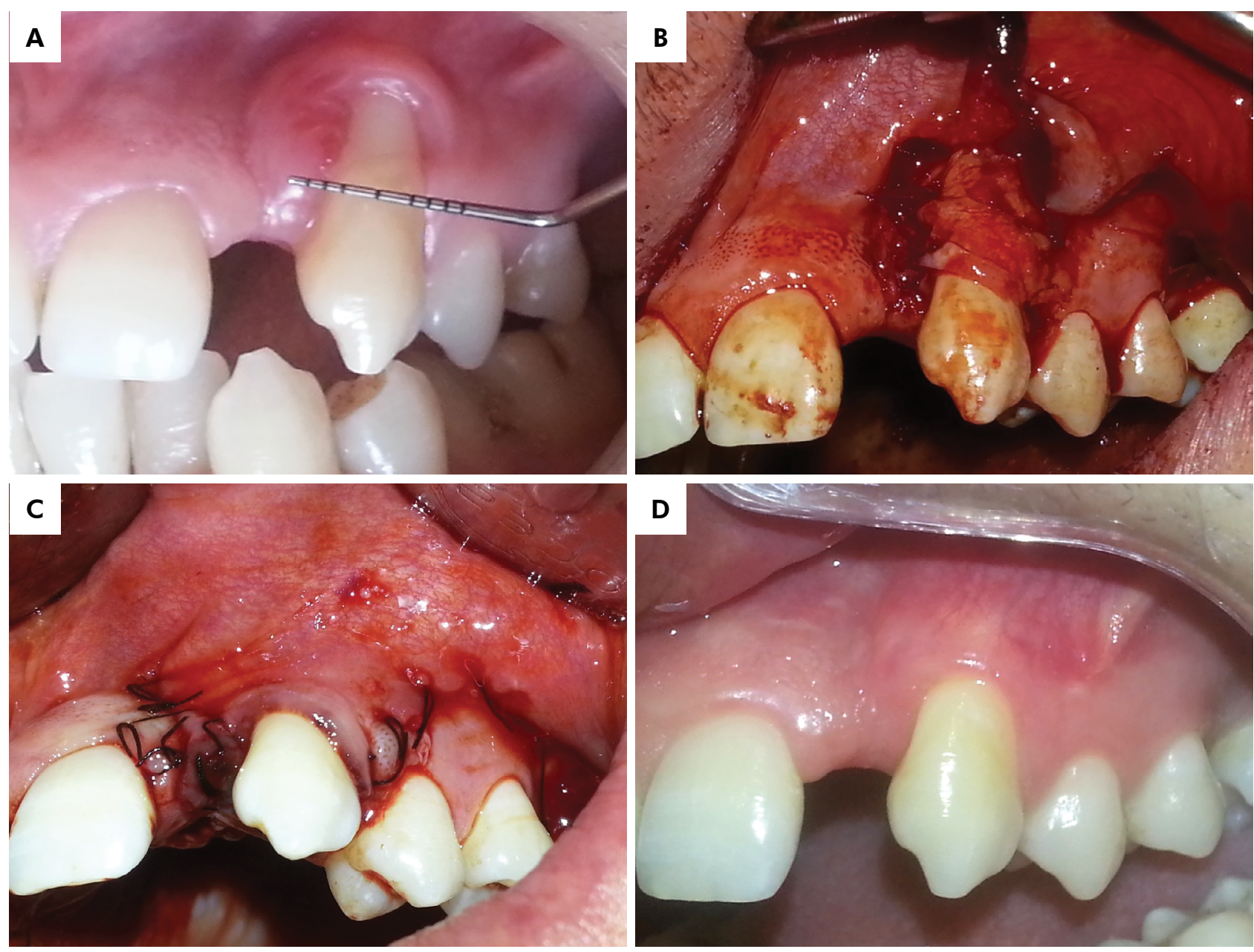

Figure 2. A. Gingival recession in left canine; B. Flap elevation and SCTG placement; C. Suturing; D. One year post-operative.

coverage aesthetic score of 10 . There was one tooth in the test group and two in the control group with a GM score of 6 but with a root coverage aesthetic score of $10>$.

Sixty-three percent of teeth in the test group and $42 \%$ of the teeth in the control group had a root coverage aesthetic score of 10 .

\section{Discussion}

In this study, SCTG+CAF and SCTG+CAF+EMD were compared in terms of root coverage using the RES system and clinical parameters in bilateral canine teeth. According to the results, despite better clinical outcomes in the EMD group, the null hypothesis that the application of EMD has no positive impact on gingival recession treatment results cannot be completely rejected. Although there was no difference between the mean RES values between the two groups, there was a significant difference between the test and control groups in terms of STT and MJA scores. These results indicate that EMD contributes to the healing of soft tissue without scarring. As a result of better wound healing, it was found that the EMD group did better than the control group in terms of the harmony of the adjacent teeth's mucogingival junction. However, the ratio of complete root coverage and RES results were found to be in favor of the test group. There was no significant difference between groups in terms of gingival recession depth and width reduction or total RES score. 
In periodontology, gingival recession treatments have typically been evaluated with complete root coverage rate or root coverage percentage. However, a reduction in gingival recession is not sufficient in itself to assess aesthetic outcomes. In evaluating the results, it was pointed out that the soft tissue should be compatible with the adjacent tissue. ${ }^{14}$ For example, a total of 38 gingival recessions were treated in this study, and 23 of them were covered completely. Of the 23 treated gingival recessions, 20 received a full score of 10 points according to the RES system. The remaining three defects did not receive 10 points because of scar formation, although the root surface was covered completely. Considering these results, it is thought that the evaluation of parameters such as MTC, STT, MJA orientation, and GC may be useful in terms of accurately conveying the outcomes. Cairo et al. ${ }^{19}$ also showed that examining only the position of the gingival margin was inadequate for evaluation of gingival recession results, and they introduced RES to cope with this restriction. Jhaveri et al. assessed the impact of acellular dermal matrix on CAF results and evaluated the aesthetic and clinical results using RES. In line with our results, they reported that although 13 of 20 gingival recessions were completely covered, only seven of them scored 10 points according to RES. ${ }^{20}$ In another study, researchers reported that after the treatment, $11 \%$ of gingival recessions had 10 points in the root coverage aesthetic scoring system, although $35 \%(n=68)$ of recessions attained complete coverage. ${ }^{14}$ It is thought in general that RES results for evaluating the wound area after periodontal surgery is a more effective method than evaluating only the root coverage. ${ }^{14,20}$

EMD application was evaluated histologically in recession defects to assess periodontal regeneration. ${ }^{21,22}$ It was reported that EMD application provided new root cementum, alveolar bone, and periodontal ligament formation when recession was treated with CAF+EMD or CAF+SCTG+EMD. ${ }^{22}$ Several studies with controversial results have used EMDs in periodontal plastic surgery for gingival recession. These studies have observed that EMDs are used in combination with different surgical methods for the treatment of gingival recessions. ${ }^{5,10,12,18,23,24}$ A summary of the results indicates that application of EMDs with CAF and / or SCTG+CAF does not result in a statistically significant difference but that EMD application affects gingival recession treatment results positively. ${ }^{18}$ A significant difference in complete root coverage rate was reported between subepithelial connective tissue grafts $(98.4 \%)$ and EMD $(71.8 \%)$ in addition to $\mathrm{CAF}^{23}$. When different study models in the literature are excluded (CAF versus CAF+EMD, CAF+EMD versus SCTG, CAF + SCTG, CAF + EMD versus CAF + CTG + EMD), we are left with three studies with which our work can be compared. ${ }^{6,12,25}$ In the first one, Roman et al. (2013) ${ }^{6}$ compared SCTG and SCTG+EMD in 38 gingival recessions; however, they studied both single and multiple gingival recessions in different teeth (incisors, canines, premolars, and molars). In our split-mouth study, all teeth were bilateral canine (19 right maxillary and 19 left maxillary canines), and multiple gingival recessions were excluded because we believed they would affect the results. The most important characteristic of the split-mouth study design, which we applied in this study, is that it eliminates a great deal of interexaminer variability from the estimates of the treatment effect. ${ }^{26}$

Like our study, Roman et al. ${ }^{6}$ reported that there is no significant difference between CAF+SCTG and $\mathrm{CAF}+\mathrm{SCTG}+\mathrm{EMD}$ in terms of RES results. However, it should be noted that RES has five variables. Although there was no difference between the total RES values between the two groups, there was a significant difference between the test and control groups in terms of STT and MJA scores. These results indicate that EMD application contributes to the healing of soft tissue without scarring. As a result of better wound healing, it was found that the EMD group did better than the control group in terms of the harmony of the mucogingival junction between adjacent teeth.

In the second study, Rasperini et al. ${ }^{25}$ compared CAF + SCTG and CAF + SCTG + EMD on 56 single gingival recession defects (15 incisors, 21 canines, and 20 premolars). The study found no significant difference between groups, but in concordance with our outcomes, the results are in favor of the EMD-applied group. In the third study, the same treatment methods as our study were applied in Miller class III gingival recessions (10 canines and 20 premolars). ${ }^{12}$ Henriques et al. reported that changes 
in the depth of gingival recession at six months and one year were statistically significant in favor of EMD. ${ }^{12}$ Our study, however, found no significant difference between groups in terms of Miller class I and class II defects.

The major limitation of the present study was its small sample size. It was hard to find bilateral gingival recessions only in maxillary canine teeth. The second important limitation was a follow-up period of one year, which is the shortest time possible for EMD follow-up. A multicentre, long-term follow-up study with a larger sample size is needed to develop further insights into EMDs and RES system validity.

\section{Conclusion}

Co-administration of EMD with SCTG was shown to affect the aesthetic scores and clinical measurements favorably, but this contribution was not found to be statistically significant for all parameters. An impact of EMD application was reported only for STT and MJA (two steps of the RES, which is composed of five variables). While there was no difference between the groups in terms of clinical measurements, we thought that this difference in tissue integration is an important point. Therefore, it can be stated that RES gives more detailed results than the root surface coverage rate used. It is thought that the RES system should be developed and used more often for evaluating gingival recession treatment outcomes.

\section{Acknowledgments}

This study was approved by Clinicaltrials.gov. Protocol registration and result system system (NCT03197610). This study was self-funded by the authors and by the University of Van Yuzuncu Yil University (Committee of Research 2014-SBE-D017). The authors thank the Faculty of Dentistry at Van Yuzuncu Yil University for its support.

\section{References}

1. Oates TW, Robinson M, Gunsolley JC. Surgical therapies for the treatment of gingival recession. A systematic review. Ann Periodontol. 2003 Dec;8(1):303-20. https://doi.org/10.1902/annals.2003.8.1.303

2. Pini-Prato G, Nieri M, Pagliaro U, Giorgi TS, La Marca M, Franceschi D, et al.; National Association of Italian Dentists (ANDI) -Tuscany Region. Surgical treatment of single gingival recessions: clinical guidelines. Eur J Oral Implantol. 2014;7(1):9-43.

3. Kassab MM, Cohen RE. The etiology and prevalence of gingival recession. J Am Dent Assoc. 2003 Feb;134(2):220-5. https://doi.org/10.14219/jada.archive.2003.0137

4. McGuire MK, Scheyer ET, Snyder MB. Evaluation of recession defects treated with coronally advanced flaps and either recombinant human platelet-derived growth factor-BB plus $\beta$-tricalcium phosphate or connective tissue: comparison of clinical parameters at 5 years. J Periodontol. 2014 Oct;85(10):1361-70. https://doi.org/10.1902/jop.2014.140006

5. Andrade PF, Grisi MF, Marcaccini AM, Fernandes PG, Reino DM, Souza SL, et al. Comparison between micro- and macrosurgical techniques for the treatment of localized gingival recessions using coronally positioned flaps and enamel matrix derivative. J Periodontol. 2010 Nov;81(11):1572-9. https://doi.org/10.1902/jop.2010.100155

6. Roman A, Soancă A, Kasaj A, Stratul SI. Subepithelial connective tissue graft with or without enamel matrix derivative for the treatment of Miller class I and II gingival recessions: a controlled randomized clinical trial. J Periodontal Res. 2013 Oct;48(5):563-72. https://doi.org/10.1111/jre.12039

7. Miron RJ, Sculean A, Cochran DL, Froum S, Zucchelli G, Nemcovsky C, et al. Twenty years of enamel matrix derivative: the past, the present and the future. J Clin Periodontol. 2016 Aug;43(8):668-83. https://doi.org/10.1111/icpe.12546

8. Huang LH, Neiva RE, Wang HL. Factors affecting the outcomes of coronally advanced flap root coverage procedure. J Periodontol. 2005 Oct;76(10):1729-34. https://doi.org/10.1902/jop.2005.76.10.1729

9. Chong $\mathrm{CH}$, Carnes DL, Moritz AJ, Oates T, Ryu OH, Simmer J, et al. Human periodontal fibroblast response to enamel matrix derivative, amelogenin, and platelet-derived growth factor-BB. J Periodontol. 2006 Jul;77(7):1242-52. https://doi.org/10.1902/jop.2006.050147

10. Rasperini G, Silvestri M, Schenk RK, Nevins ML. Clinical and histologic evaluation of human gingival recession treated with a subepithelial connective tissue graft and enamel matrix derivative (Emdogain): a case report. Int J Periodontics Restorative Dent. 2000 Jun;20(3):269-75. 
11. Sculean A, Windisch P, Keglevich T, Gera I. Histologic evaluation of human intrabony defects following non-surgical periodontal therapy with and without application of an enamel matrix protein derivative. J Periodontol. 2003 Feb;74(2):153-60. https://doi.org/10.1902/jop.2003.74.2.153

12. Henriques PS, Pelegrine AA, Nogueira AA, Borghi MM. Application of subepithelial connective tissue graft with or without enamel matrix derivative for root coverage: a split-mouth randomized study. J Oral Sci. 2010 Sep;52(3):463-71. https://doi.org/10.2334/josnusd.52.463

13. Bosshardt DD. Biological mediators and periodontal regeneration: a review of enamel matrix proteins at the cellular and molecular levels. J Clin Periodontol. 2008 Sep;35(8 Suppl):87-105. https://doi.org/10.1111/j.1600-051X.2008.01264.x

14. Pini-Prato G. The Miller classification of gingival recession: limits and drawbacks. J Clin Periodontol. 2011 Mar;38(3):243-5. https://doi.org/10.1111/j.1600-051X.2010.01655.x

15. Cairo F, Pagliaro U, Nieri M. Treatment of gingival recession with coronally advanced flap procedures: a systematic review. J Clin Periodontol. 2008 Sep;35(8 Suppl):136-62. https://doi.org/10.1111/j.1600-051X.2008.01267.x

16. Cairo F, Rotundo R, Miller PD Jr, Pini Prato GP. Root coverage esthetic score: a system to evaluate the esthetic outcome of the treatment of gingival recession through evaluation of clinical cases. J Periodontol. 2009 Apr;80(4):705-10. https://doi.org/10.1902/jop.2009.080565

17. Pilloni A, Paolantonio M, Camargo PM. Root coverage with a coronally positioned flap used in combination with enamel matrix derivative: 18-month clinical evaluation. J Periodontol. 2006 Dec;77(12):2031-9. https://doi.org/10.1902/jop.2006.050390

18. McGuire MK, Scheyer ET, Nunn M. Evaluation of human recession defects treated with coronally advanced flaps and either enamel matrix derivative or connective tissue: comparison of clinical parameters at 10 years. J Periodontol. 2012 Nov;83(11):1353-62. https://doi.org/10.1902/jop.2012.110373

19. Cairo F, Pagliaro U, Buti J, Baccini M, Graziani F, Tonelli P, et al. Root coverage procedures improve patient aesthetics. A systematic review and Bayesian network meta-analysis. J Clin Periodontol. 2016 Nov;43(11):965-75. https://doi.org/10.1111/icpe.12603

20. Jhaveri HM, Chavan MS, Tomar GB, Deshmukh VL, Wani MR, Miller PD Jr. Acellular dermal matrix seeded with autologous gingival fibroblasts for the treatment of gingival recession: a proof-of-concept study. J Periodontol. 2010 Apr;81(4):616-25. https://doi.org/10.1902/jop.2009.090530

21. Heijl L. Periodontal regeneration with enamel matrix derivative in one human experimental defect. A case report. J Clin Periodontol. 1997 Sep;24(9 P+ 2):693-6.

22. McGuire MK, Cochran DL. Evaluation of human recession defects treated with coronally advanced flaps and either enamel matrix derivative or connective tissue. Part 2: histological evaluation. J Periodontol. 2003 Aug;74(8):1126-35. https://doi.org/10.1902/jop.2003.74.8.1126

23. Zuhr O, Rebele SF, Schneider D, Jung RE, Hürzeler MB. Tunnel technique with connective tissue graft versus coronally advanced flap with enamel matrix derivative for root coverage: a RCT using 3D digital measuring methods. Part I. Clinical and patient-centred outcomes. J Clin Periodontol. 2014 Jun;41(6):582-92. https://doi.org/10.1111/jcpe.12178

24. Rebele SF, Zuhr O, Schneider D, Jung RE, Hürzeler MB. Tunnel technique with connective tissue graft versus coronally advanced flap with enamel matrix derivative for root coverage: a RCT using 3D digital measuring methods. Part II. Volumetric studies on healing dynamics and gingival dimensions. J Clin Periodontol. 2014 Jun;41(6):593-603. https://doi.org/10.1111/jcpe.12254

25. Rasperini G, Roccuzzo M, Francetti L, Acunzo R, Consonni D, Silvestri M. Subepithelial connective tissue graft for treatment of gingival recessions with and without enamel matrix derivative: a multicenter, randomized controlled clinical trial [Internet]. Int J Periodontics Restorative Dent. 2011 Apr;31(2):133-9.

26. Lesaffre E, Philstrom B, Needleman I, Worthington $H$. The design and analysis of split-mouth studies: What statisticians and clinicians should know. Stat Med. 2009 Dec 10 [cited 2018 Mar 5];28(28):3470-82. 